\title{
Vulnerabilidade e fatores de risco associados para Covid-19 em idosos institucionalizados
}

\author{
Vulnerability and associated risk factors for Covid-19 in institutionalized elderly \\ Vulnerabilidad y factores de riesgo asociados para Covid-19 en personas \\ institucionalizadas
}

Talita Araujo de Souza ${ }^{1 *}$, Vilani Medeiros de Araújo Nunes ${ }^{1}$, Izabel Cristina Santos do Nascimento ${ }^{1}$, Luylla Astéria Maia Delmiro', Miria Mendonça de Morais ${ }^{1}$, Thaiza Teixeira Xavier Nobre ${ }^{1}$, Luciana Araújo dos Reis ${ }^{2}$, Ana Elza Oliveira de Mendonça ${ }^{1}$, Gilson de Vasconcelos Torres ${ }^{1}$.

\section{RESUMO}

Objetivo: Identificar a associação de fatores de risco para COVID-19 com a vulnerabilidade em pessoas idosas residentes em instituições de longa permanência. Métodos: Trata-se de um estudo descritivo, exploratório, com abordagem quantitativa, realizado em oito Instituições de Longa Permanência para Idosos (ILPI) na região metropolitana de Natal, Rio Grande do Norte, Brasil. Foram incluídos idosos com idade igual ou superior a 60 anos, que estivessem presentes na instituição durante a coleta das informações. Na obtenção dos dados foi utilizado o protocolo de identificação do idoso vulnerável inserido na Caderneta de Saúde da Pessoa Idosa. Para análise utilizou-se o software SPSS versão 21.0 e aplicados testes Qui-quadrado de Pearson e Razão de Chance. Resultados: Verificou-se que houve associação do número de itens de vulnerabilidade (até 3 itens) nos idosos que apresentaram até 3 fatores de risco para COVID-19 ( $p=0,008)$, com razão de chance de 2,2. Conclusão: A predominância de fatores de risco para COVID-19 indica maior vulnerabilidade à evolução da forma grave da COVID-19, associada ao maior risco de mortalidade, o que evidencia necessidade do monitoramento da saúde dos idosos.

Palavras-chave: Envelhecimento, Infecções por coronavirus, Instituição de longa permanência para idosos, Pessoa idosa, Institucionalização.

\section{ABSTRACT}

Objective: To identify the association of risk factors for COVID-19 with vulnerability in elderly people living in long-term care facilities. Methods: This is a descriptive, exploratory study, with a quantitative approach, carried out in eight LTCFs in the metropolitan region of Natal, Rio Grande do Norte, Brazil. Elderly people aged 60 years or over, able to participate in the study were included. In order to obtain the data, the identification protocol for vulnerable elderly people used in the Health Record of the Elderly was used. For analysis, SPSS software version 21.0 was used and Pearson's Chi-square and Chance Ratio tests were applied. Results: It was found that there was an association of the number of vulnerability items (up to 3 items) in the elderly who presented up to 3 risk factors for COVID-19 ( $p=0.008)$, with a odds ratio of 2.2. Conclusion: The predominance of risk factors for COVID-19 indicates greater vulnerability to the evolution of the severe form of CO-VID-19, associated with a higher risk of mortality, which evidences the need for monitoring the health of the elderly.

Keywords: Aging, Coronavirus, Coronavirus infections, Long-term institution for the elderly, Aged, Institutionalization.

\section{RESUMEN}

Objetivo: Identificar la asociación de factores de riesgo para COVID-19 con la vulnerabilidad en personas mayores que viven en centros de atención a largo plazo. Métodos: Este es un estudio descriptivo, exploratorio, con un enfoque cuantitativo, llevado a cabo en ocho LTCF en la región metropolitana de Natal, Rio Grande do Norte, Brasil. Se incluyeron personas mayores de 60 años o más que podían participar en el estudio. Para obtener los datos, se utilizó el protocolo de identificación de personas mayores vulnerables que

1Universidade Federal do Rio Grande do Norte (UFRN), Natal - RN. *E-mail: talitaaraujo23@hotmail.com 2Universidade Estadual do Sudoeste da Bahia (UESB), Jequié - BA. 
se utiliza en el Registro de Salud de los Ancianos. Se usó el software SPSS versión 21.0 para el análisis y se aplicaron las pruebas de Chi-cuadrado y Ratio de probabilidad de Pearson. Resultados: Se encontró que había una asociación del número de ítems de vulnerabilidad (hasta 3 ítems) en los ancianos que presentaron hasta 3 factores de riesgo para COVID-19 $(p=0.008)$, con una razón de probabilidades de 2.2. Conclusión: El predominio de los factores de riesgo para COVID-19 indica una mayor vulnerabilidad a la evolución de la forma grave de COVID-19, asociada con un mayor riesgo de mortalidad, lo que destaca la necesidad de controlar la salud de los ancianos.

Palabras clave: Envejecimiento, Infecciones por coronavirus, COVID-19, Institución de larga duración para personas mayores, Anciano, Institucionalización.

\section{INTRODUÇÃO}

A COVID-19 (Corona Virus Disease-19) é uma doença nova causada pelo Coronavírus Severe Acute Respiratory Syndrome Coronavirus-2 (SARS-CoV-2) (AGÊNCIA NACIONAL DE VIGILÂNCIA SANITÁRIA, 2020) cujo quadro clínico varia de infecções assintomáticas a quadros respiratórios graves. De acordo com a Organização Mundial de Saúde (OMS), a maioria dos pacientes com COVID-19 (cerca de 80\%) podem ser assintomáticos e $20 \%$ dos casos podem requerer atendimento hospitalar por apresentarem dificuldade respiratória e desses casos aproximadamente $5 \%$ podem necessitar de suporte para o tratamento de insuficiência respiratória (suporte ventilatório) (ZHANG W, 2020).

As infecções por Coronavirus geralmente apresentam sintomas leves, limitados ou inexistentes e, portanto, não são reconhecidas (MINISTÉRIO DA SAÚDE, 2020). Em consequência, a depender de sua contagiosidade e número, podem expor uma parcela muito maior da população ao vírus, principalmente as pessoas do grupo de risco, integrado por pessoas idosas e de qualquer idade que sejam imunodeprimidas, cardiopatas, diabéticas, hipertensas, ou que tenham doenças pré-existentes crônicas respiratórias, nas quais as taxas de letalidade são bem maiores (PETRILLI CM, et al., 2020).

Nesse sentido, uma das grandes preocupações acerca da pandemia do novo coronavírus é a maior vulnerabilidade de alguns grupos e estratos da população aos efeitos da doença respiratória COVID-19. Para a comunidade científica, é consenso que a pessoa idosa e portadores de doenças crônicas apresentam um perfil mais suscetível diante da infecção, o que já foi documentado em diferentes estudos e pesquisas realizados em todo mundo (LIMA DLF, et al., 2020).

Outra inquietação envolve os idosos residentes em Instituição de Longa Permanência para Idosos (ILPI), pois estudos preliminares apontam que, nestas realidades, a infecção pelo SARS-CoV-2 é alta, com sugestão de taxa de mortalidade para maiores de 80 anos, superior a 15\% (OXFORD COVID-19 EVIDENCE SERVICE, 2020). Este contexto é considerado de alto risco para infecção, pois envolve predominantemente idosos, diversos com comorbidades crônicas e dificuldades para atividades da vida diária; contato frequente de cuidadores, profissionais e visitantes; e convivência em aglomerados (JESUS IT, et al., 2017).

As Instituições de Longa Permanência para Idosos representam um forte e principal fator de risco para o aumento da morbidade e mortalidade pelo COVID-19, pois, possui características inerentes ao vírus que se configuram como condições necessárias para o acometimento. Países da América do Norte, Oceania e Europa de todos os óbitos ocorridos pelo COVID-19, entre 30\% e 60\% ocorreram em locais como ILPI e afins (TAN LF, SEETHARAMAN SK, 2020).

A pandemia da COVID-19 fez com que os cuidados nas ILPI fossem acentuados. Para o controle dessa doença as medidas preventivas são as estratégias mais efetivas para diminuição do risco e contaminação dos idosos residentes em ILPI, sendo: restrição de visitas, monitoramento do controle de entrada e saída dos trabalhadores e prestadores de serviços, maior exigência com lavagem de mãos e utilização continua de álcool a 70\%, rastreamento e monitoramento da presença de síndromes gripais, alertando acerca dos sintomas de febre e sintomas respiratórios, troca de roupas e calçados, além do controle rigoroso dos alimentos que entram no local. O uso de máscara facial é recomendado para todos os trabalhadores das ILPI e também para os idosos residentes quando exercem tarefas fora dos seus quartos. 
Além disso, medidas como distanciamento social, separação maior entre os trabalhadores e idosos em tarefas relacionadas ao cuidado, diminuição de atividades em grupo e refeições coletivas são medidas que devem ser adotadas (TAN LF, SEETHARAMAN SK, 2020).

No que se refere ao processo de institucionalização, a vulnerabilidade torna-se mais aumentada tendo em vista o confinamento social onde em muitas realidades os idosos encontram-se em ambientes coletivos levando-os ao aumento no risco de adoecerem com comorbidades em espaços coletivos, tornando-os mais suscetíveis a contraírem doenças infecto contagiosas (BENKSIM A, et al., 2020).

Diante desse contexto e tendo em vista os aspectos presentes da vulnerabilidade na faixa etária de pessoas com idade igual e acima de 60 anos, o presente estudo teve como objetivo identificar a associação de fatores de risco para COVID-19 com a vulnerabilidade em pessoas idosas residentes em instituições de longa permanência.

\section{MÉTODOS}

Estudo descritivo, exploratório, com abordagem quantitativa, desenvolvido em oito ILPI nas cidades de Natal, Parnamirim e Macaíba, localizadas no Estado do Rio Grande do Norte - RN. Trata-se de instituições de caráter filantrópico, sem fins lucrativos, com capacidade física e profissional para atender idosos em seus diversos graus de dependência, conforme determina RDC n² 283/05 MS/ANVISA, estabelecendo padrões mínimos de funcionamento das instituições.

Foram estabelecidos como critérios de inclusão pessoas com idade igual ou superior a 60 anos, residentes em ILPI sem fins lucrativos das localidades descritas e que estivessem presentes durante a aplicação do instrumento de coleta dos dados (Caderneta de Saúde da Pessoa Idosa). Foram excluídos os idosos que não estavam presentes nas instituições no momento da coleta. Após a aplicação dos critérios de inclusão e exclusão a população do estudo foi representada por 268 idosos institucionalizados.

A coleta dos dados ocorreu entre os meses de fevereiro e dezembro de 2018 como parte do estudo intitulado "Monitorando a Saúde da Pessoa Idosa" da Universidade Federal do Rio Grande do Norte. Os dados dos idosos foram obtidos a partir das informações contidas na Caderneta de Saúde da Pessoa Idosa considerada um instrumento estratégico que permite auxiliar e acompanhar a saúde dos idosos por meio do registro periódico de cinco anos, contendo informações socioeconômicas, familiares, das condições de saúde, hábitos de vida, identificação de idosos vulneráveis, avaliação nutricional etc.

Participaram da coleta de dados uma equipe multiprofissional composta de enfermeiras, fisioterapeutas, nutricionistas e cirurgiões dentistas. Todos os participantes foram qualificados no contexto de uma oficina de formação cujo objetivo foi alinhar a equipe em relação a utilização da caderneta preconizada pelo Ministério da Saúde.

Para a identificação do idoso vulnerável utilizou-se as informações do Vulnerable Elders Survey (VES-13) que é uma ferramenta com uma estrutura simples e de fácil aplicabilidade na qual é possível identificar quais as variáveis com forte associação ao risco de declínio funcional como: idade, autopercepção de saúde, presença de limitação física e incapacidade funcional. No total, há 13 questões autorreferidas e objetivas para as quais foram atribuídos escores. Idosos com pontuação menor ou igual a 03 são classificados como não vulneráveis; já a pontuação superior a 03 indica vulnerabilidade. A referida ferramenta encontra-se inserida na Caderneta de Saúde da Pessoa Idosa (BRASIL, 2013).

Os dados foram organizados em planilhas no software Microsoft Excel 2010, e posteriormente analisados com o auxílio do programa IBM SPSS Statistic versão 21.0 for Windows, sendo aplicados os testes Quiquadrado de Pearson e Razão de Chance.

O presente projeto de pesquisa foi submetido ao Comitê de Ética em Pesquisa do Hospital Onofre Lopes (CEP/HUOL) da Universidade Federal do Rio Grande do Norte, sendo aprovado sob CAAE 78891717.7.0000.5292 e parecer $\mathrm{n}$-2 2.366.555. Todos os participantes assinaram um Termo de Consentimento Livre e Esclarecido. 


\section{RESULTADOS}

Constatou-se no presente estudo uma maior frequência do sexo feminino $(69,3 \%)$, idade acima de 80 anos $(54,3 \%)$, solteiros $(49,2 \%)$, sem nenhuma escolari-dade $(39,0 \%)$ e de raça/cor não branca $(51,2 \%)$. Quanto aos hábitos de vida, verificou-se uma maior distribuição dos não tabagistas $(87,7 \%)$ e que não consomem álcool (94,4\%). Observou-se na tabela 1 que os fatores de risco para COVID-19 que apresentaram maior frequência foram Índice de Massa Corporal (IMC) alterado $(64,8 \%$; $p$-valor $<0,001)$, deficiência cognitiva $(57,3 \%$; p-valor $=0,017)$ e hipertensão $(56,6 \%$; $p$-valor=0,032) (Tabela 1).

Tabela 1 - Fatores de Risco para COVID-19 em pessoas idosas residentes em instituições de longa permanência.

\begin{tabular}{|c|c|c|c|c|}
\hline \multirow{2}{*}{\multicolumn{2}{|c|}{ Fatores de risco COVID19 }} & \multicolumn{2}{|c|}{ Idosos - ILPI } & \multirow{2}{*}{$\begin{array}{l}\text { Qui-quadrado } \\
\text { (p-valor) }\end{array}$} \\
\hline & & $\mathrm{n}$ & $\%$ & \\
\hline \multirow{2}{*}{ IMC alterado } & Sim & 173 & 64,8 & \multirow{2}{*}{$<0,001$} \\
\hline & Não & 94 & 35,2 & \\
\hline \multirow{2}{*}{ Hipertensão } & Sim & 151 & 56,6 & \multirow{2}{*}{0,032} \\
\hline & Não & 116 & 43,4 & \\
\hline \multirow{2}{*}{ Diabetes } & Sim & 73 & 27,3 & \multirow{2}{*}{$<0,001$} \\
\hline & Não & 194 & 72,7 & \\
\hline \multirow{2}{*}{ Tabagismo } & Sim & 35 & 13,1 & \multirow{2}{*}{$<0,001$} \\
\hline & Não & 232 & 86,9 & \\
\hline \multirow{2}{*}{ Doença coronária } & Sim & 15 & 5,6 & \multirow{2}{*}{$<0,001$} \\
\hline & Não & 252 & 94,4 & \\
\hline \multirow{2}{*}{ Asma } & Sim & 7 & 2,6 & \multirow{2}{*}{$<0,001$} \\
\hline & Não & 260 & 97,4 & \\
\hline \multirow{2}{*}{ DPOC } & Sim & 6 & 2,2 & \multirow{2}{*}{$<0,001$} \\
\hline & Não & 261 & 97,8 & \\
\hline
\end{tabular}

Legenda: ILPI: Instituições de Longa Permanência; IMC: Índice de Massa Corporal; DPOC: Doença Pulmonar Obstrutiva Crônica.

Fonte: Souza TA, et al., 2020. Dados da pesquisa, 2018.

Analisando os itens de vulnerabilidade da tabela 2 , observou-se que a incapacidade $(87,6 \%)$, limitação física $(80,9 \%)$ e faixa etária vulnerável $(71,5 \%)$ estiveram presentes com significância estatística $(p<0,001)$. A vulnerabilidade estava presente em $83,1 \%(p<0,001)$, tendo a incapacidade, limitação física e faixa etária vulnerável, como itens mais importantes na determinação da vulnerabilidade (Tabela 2).

Tabela 2 - Itens de vulnerabilidade (VES-13) de idosos residentes em instituições de longa permanência nos municípios pesquisados.

\begin{tabular}{|c|c|c|c|c|}
\hline \multicolumn{2}{|c|}{ Itens de vulnerabilidade (VES-13) } & \multicolumn{2}{|c|}{ Idosos - ILPI } & \multirow{2}{*}{ Qui-quadrado (p-valor) } \\
\hline & & $\mathbf{n}$ & $\%$ & \\
\hline Incapacidade & $\begin{array}{l}\text { Presente } \\
\text { Ausente }\end{array}$ & $\begin{array}{c}234 \\
33\end{array}$ & $\begin{array}{l}87,6 \\
12,4\end{array}$ & $<0,001$ \\
\hline Limitação física & $\begin{array}{l}\text { Presente } \\
\text { Ausente }\end{array}$ & $\begin{array}{c}216 \\
51\end{array}$ & $\begin{array}{l}1<, 4 \\
80,9 \\
19,1\end{array}$ & $<0,001$ \\
\hline Faixa etária & $\begin{array}{l}\text { Presente } \\
\text { Ausente }\end{array}$ & $\begin{array}{l}191 \\
76\end{array}$ & $\begin{array}{l}71,5 \\
28,5\end{array}$ & $<0,001$ \\
\hline Autopercepção da saúde & $\begin{array}{l}\text { Presente } \\
\text { Ausente }\end{array}$ & $\begin{array}{l}113 \\
154\end{array}$ & $\begin{array}{l}42,3 \\
57,7\end{array}$ & 0,012 \\
\hline Vulnerabilidade & $\begin{array}{l}\text { Presente } \\
\text { Ausente }\end{array}$ & $\begin{array}{c}222 \\
45\end{array}$ & $\begin{array}{l}83,1 \\
16,9\end{array}$ & $<0,001$ \\
\hline
\end{tabular}

Legenda: ILPI: Instituições de Longa Permanência.

Fonte: Souza TA, et al., 2020.

Os dados da Tabela 3 apresentam os fatores de risco da COVID-19 com a presença de itens de vulnerabilidade dos idosos residentes em ILPI. Observou-se que houve associação significativa nos idosos com IMC alterado com os fatores de incapacidade, limitação física, faixa etária com $p<0,001$ e vulnerabilidade total $p=0,001$. A presença da deficiência cognitiva mostrou-se com diferenças significantes na incapacidade 
( $p=0,009)$, limitação física $(p=0,014)$, percepção de saúde $(p=0,0,48)$ e vulnerabilidade total $(p=0,016)$. A presença de hipertensão foi significante com os itens faixa etária $(p=0,025)$, percepção de saúde $(p=0,006)$ e vulnerabilidade total $(p=0,044)$.

No que diz respeito a faixa etária como fator de risco, houve associação significante nos itens de vulnerabilidade, faixa etária $(p<0,001)$ e vulnerabilidade total $(p=0,016)$. A ausência de tabagismo, doença coronariana, asma e DPOC, mostraram-se como diferença significante em todos os fatores de vulnerabilidade com $p<0,001$ (Tabela 3).

Tabela 3 - Associação dos Fatores COVID-19 com a presença dos itens de vulnerabilidade em idosos residentes em instituições de longa permanência nos municípios pesquisados.

\begin{tabular}{|c|c|c|c|c|c|}
\hline \multirow[b]{2}{*}{ Fatores de COVID19 } & \multicolumn{5}{|c|}{ Presença dos itens e total de vulnerabilidade em idosos } \\
\hline & $\begin{array}{c}\text { Incapacidade } \\
\text { n (\%) }\end{array}$ & $\begin{array}{c}\text { Limitação física } \\
\text { n (\%) }\end{array}$ & $\begin{array}{c}\text { Faixa etária } \\
\text { n (\%) }\end{array}$ & $\begin{array}{l}\text { Percepção de } \\
\text { saúde n (\%) }\end{array}$ & $\begin{array}{c}\text { Vulnerabilidade } \\
\text { Total n (\%) }\end{array}$ \\
\hline \multicolumn{6}{|l|}{ IMC Alterado } \\
\hline Presente & $151(64,5)$ & $135(62,5)$ & $125(65,4)$ & $64(56,6)$ & $139(62,6)$ \\
\hline Ausente & $83(35,5)$ & $81(37,5)$ & $66(34,6)$ & $49(43,4)$ & $83(37,4)$ \\
\hline p-valor & $<0,001$ & $<0,001$ & $<0,001$ & 0,158 & 0,001 \\
\hline \multicolumn{6}{|l|}{ Deficiência cognitiva } \\
\hline Presente & $137(58,5)$ & $126(58,3)$ & $105(55,0)$ & $67(59,3)$ & $129(58,1)$ \\
\hline Ausente & $97(41,5)$ & $90(41,7)$ & $86(45,0)$ & $46(40,7)$ & $93(41,9)$ \\
\hline p-valor & 0,009 & 0,014 & 0,169 & 0,048 & 0,016 \\
\hline \multicolumn{6}{|l|}{ Hipertensão } \\
\hline Presente & $130(56,6)$ & $122(56,5)$ & $111(58,1)$ & $71(62,8)$ & $126(56,8)$ \\
\hline Ausente & $104(44,4)$ & $94(43,5)$ & $80(41,9)$ & $42(37,2)$ & $96(43,2)$ \\
\hline p-valor & 0,089 & 0,057 & 0,025 & 0,006 & 0,044 \\
\hline \multicolumn{6}{|l|}{ Faixa etária } \\
\hline Presente & $131(56,0)$ & $121(56,0)$ & $133(69,6)$ & $54(47,8)$ & $129(58,1)$ \\
\hline Ausente & $103(44,0)$ & $95(44,0)$ & $58(30,4)$ & $59(52,2)$ & $93(41,9)$ \\
\hline p-valor & 0,067 & 0,077 & $<0,001$ & 0,638 & 0,016 \\
\hline \multicolumn{6}{|l|}{ Diabetes } \\
\hline Presente & $62(26,5)$ & $60(27,8)$ & $49(25,7)$ & $38(33,6)$ & $62(27,9)$ \\
\hline Ausente & $172(73,5)$ & $156(72,2)$ & $142(74,3)$ & $75(66,4)$ & $160(72,1)$ \\
\hline $\mathrm{p}$-valor & $<0,001$ & $<0,001$ & $<0,001$ & 0,001 & $<0,001$ \\
\hline \multicolumn{6}{|l|}{ Tabagismo } \\
\hline Presente & $29(12,4)$ & $21(9,7)$ & $24(12,6)$ & $13(11,5)$ & $25(11,3)$ \\
\hline Ausente & $205(87,6)$ & $195(90,3)$ & $167(87,4)$ & $100(88,5)$ & $197(88,7)$ \\
\hline$p$-valor & $<0,001$ & $<0,001$ & $<0,001$ & $<0,001$ & $<0,001$ \\
\hline \multicolumn{6}{|l|}{ Doença coronária } \\
\hline Presente & $14(6,0)$ & $16(7,4)$ & $13(2,1)$ & $5(4,4)$ & $3(1,4)$ \\
\hline Ausente & $220(94,0)$ & $200(92,6)$ & $172(97,9)$ & $103(95,6)$ & $219(98,6)$ \\
\hline $\mathrm{p}$-valor & $<0,001$ & $<0,001$ & $<0,001$ & $<0,001$ & $<0,001$ \\
\hline \multicolumn{6}{|l|}{ Asma } \\
\hline Presente & $4(1,7)$ & $6(2,8)$ & $4(4,4)$ & $5(4,4)$ & $4(1,8)$ \\
\hline Ausente & $230(98,3)$ & $210(97,2)$ & $187(95,6)$ & $108(95,6)$ & $218(98,2)$ \\
\hline $\mathrm{p}$-valor & $<0,001$ & $<0,001$ & $<0,001$ & $<0,001$ & $<0,001$ \\
\hline \multicolumn{6}{|l|}{ DPOC } \\
\hline Presente & $3(2,3)$ & $5(2,3)$ & $2(1,0)$ & $3(2,7)$ & $3(1,4)$ \\
\hline Ausente & $231(98,7)$ & $211(97,7)$ & $189(99,0)$ & $110(97,7)$ & $219(98,6)$ \\
\hline$p$-valor & $<0,001$ & $<0,001$ & $<0,001$ & $<0,001$ & $<0,001$ \\
\hline Total & $234(100,0)$ & $216(100,0)$ & $191(100,0)$ & $113(100,0)$ & $222(100,0)$ \\
\hline
\end{tabular}

Legenda: ILPI: Instituições de Longa Permanência; IMC: Índice de Massa Corporal; DPOC: Doença Pulmonar Obstrutiva Crônica. Fonte: Souza TA, et al., 2020. 
Verificou-se que houve associação do número de itens de vulnerabilidade (até 3 itens) nos idosos que apresentaram até 3 fatores de risco para COVID-19 ( $p=0,008)$, com razão de chance de 2,2 (Tabela 4).

Tabela 4 - Associação do número de fatores COVID-19 com o número de itens de vulnerabilidade em idosos residentes em instituições de longa permanência nos municípios pesquisados.

\begin{tabular}{|c|c|c|c|c|}
\hline \multirow{2}{*}{$\begin{array}{l}\text { Fatores COVID19 } \\
\text { associados }\end{array}$} & \multicolumn{2}{|c|}{$\begin{array}{l}\text { Itens de vulnerabilidade } \\
\text { associados }\end{array}$} & \multirow{2}{*}{$\begin{array}{l}\text { Total } \\
\text { n (\%) }\end{array}$} & \multirow{2}{*}{$\begin{array}{c}\text { Qui-quadrado } \\
\text { p-valor } \\
\text { RC (IC95\%) }\end{array}$} \\
\hline & $\begin{array}{c}\text { Até } 3 \text { itens } \\
\text { n (\%) }\end{array}$ & $\begin{array}{c}>3 \text { itens } \\
\text { n (\%) }\end{array}$ & & \\
\hline Até 3 fatores & $160(59,9)$ & $39(14,6)$ & $199(74,5)$ & \multirow{3}{*}{$\begin{array}{c}0,008 \\
2,2(1,2-4,1)\end{array}$} \\
\hline$>3$ fatores & $44(20,4)$ & $24(9,0)$ & $68(25,5)$ & \\
\hline Total & $204(76,4)$ & $63(23,6)$ & $267(100,0)$ & \\
\hline
\end{tabular}

Fonte: Souza TA, et al., 2020.

\section{DISCUSSÃO}

Um dos grandes destaques acerca da pandemia do COVID-19 é a sua associação com a vulnerabilidade em pessoas idosas. O envelhecimento está diretamente associado ao declínio natural das funções fisiológicas, repercutindo no cotidiano do indivíduo que a vivência, resultando em um maior acúmulo de doenças crônicas, além de resultar no desenvolvimento da vulnerabilidade (ARAÚJO JRFB et al., 2019).

Estudo feito a nível de Brasil com dados até 25 de maio de 2020, mostrou que o estado do Rio Grande do Norte tinha 16,54\% casos de COVID-19 em idosos (BARBOSA IR, et al., 2020) o que mostra que o transcurso da doença estava até então atingindo pessoas acima de 60 anos. No presente estudo verificou-se uma maior frequência de idosos classificados como vulneráveis, dados estes corroborados por estudo realizado em uma ILPI de São Paulo/SP sobre vulnerabilidade utilizando o VES-13, no qual $67,3 \%(n=35)$ dos idosos da amostra total apresentaram vulnerabilidade (BROOKE J e DEBRA J, 2020).

Ser idoso é considerado um fator de risco para a contaminação da COVID-19, como mostra um estudo realizado em dois hospitais na cidade de Wuhan na China em dezembro de 2019, que identificou o aumento da idade está associado a maior vulnerabilidade em contrair a doença e a maior mortalidade visto que, a gravidade da doença torna-se maior nesse público. Quanto mais vulnerável for o idoso maior seu risco para contrair a COVID-19 na condição mais grave12. Este quadro ainda se agrava ainda mais em idosos residentes em ILPI devido ao fato destes apresentarem esses e outros fatores de riscos obtidos nos resultados, ainda de forma associada (NUNES VM, et al., 2020; LLOYD-SHERLOCK P, et al., 2020; PEREIRA FM, et al., 2020; GUTHS JCFS, et al., 2017).

Alguns fatores se destacaram importantes para determinar a vulnerabilidade no presente estudo, como a incapacidade, limitação física e faixa etária. A incapacidade funcional exige habilidades complexas para se conviver em sociedade. Uma vez que ocorre o processo de institucionalização há também mudanças no cotidiano do idoso, no qual vai interferir no desenvolvimento de ações socioeconômica e familiares, como é o caso das Atividades Instrumentais da Vida Diária (AIVD) onde o idoso é capaz de realizar compras, controlar suas finanças, realizar tarefas domésticas leves, tomar banho sozinho e caminhar. Nesse contexto, a incapacidade acarreta danos na qualidade de vida da pessoa idosa (CRUZ RR, et al., 2019).

A incapacidade funcional influência diretamente nas limitações físicas implicando em dificuldades na realização de determinadas atividades cotidianas, como: curvar-se, ajoelhar-se, levantar ou carregar objetos com aproximadamente cinco quilos, escrever ou manusear pequenos objetos e elevar ou estender os braços acima do nível do ombro. Essas limitações na população idosa residente nas instituições de longa permanência para idosos, a torna cada vez mais sedentária levando a danos nas condições de saúde, onde há o comprometimento da capacidade cognitiva, o aumento de comorbidades e a perda da autonomia (BARBOSA KTF, et al., 2017). 
Em relação a funcionalidade a maioria dos idosos avaliados foram classificados com incapacidade funcional. Dados estes semelhantes ao de estudo realizado em Santa Catarina utilizando dados da Pesquisa Nacional de Saúde que mostrou prevalência total de incapacidade de 15,9\% nas Atividades Básicas de Vida Diária (ABVD) e 29,9\%. Atividades Instrumentais de Vida Diária (AIVD). É admissível afirmar que essas variáveis estão contidas em uma rede multifatorial está associada à incapacidade funcional no idoso. Essa rede apresenta fatores ligados principalmente a hábitos de vida saudável e sua prevenção passa necessariamente por mudanças de comportamento (SCHMIDT TP, 2019).

A faixa etária entre 75 ou mais possui maior risco de vulnerabilidade, visto que o avançar da idade contribui significativamente para o surgimento e/ou aumento significativo de processos patológicos, da mesma maneira que aumenta a dificuldade para a realização de atividades básicas e instrumentais da vida diária, gerando uma maior dependência para a manutenção do autocuidado e o bem-estar (SILVA RS, 2019).

Assim, os idosos são considerados um fator de preocupação para a contaminação com COVID-19, uma vez que o aumento da idade está associado à morte. O risco de mortalidade pela infecção viral do COVID-19 aumenta com o avançar da idade e se agrava com a pré-existência de comorbidades como diabetes, hipertensão e doenças cardiovasculares, dessa forma, atinge principalmente a população idosa (ORGANIZAÇÃO PAN-AMERICANA DE SAÚDE, 2020).

O risco de morte por COVID-19 aumenta com a idade e a maioria das mortes observadas ocorre em pessoas com mais de 60 anos, especialmente naquelas com condições crônicas, como doenças cardiovasculares. Isso tem implicações importantes para a maneira pela qual a saúde pública e as respostas clínicas devem ser desenvolvidas (LLOYD-SHERLOCK P, et al., 2020)

A presença de IMC alterado, a deficiência cognitiva e a hipertensão na maior parte dos idosos avaliados em foram os fatores de risco para COVID-19 mais importantes nos idosos pesquisados. A identificação de fatores de risco para o aumento da gravidade no contágio com o vírus é essencial (PEREIRA FM, et al., 2020). A partir da classificação dos fatores de riscos é possível identificar os idosos com maior probabilidade para prognóstico desfavorável à infecção, norteando a priorização das medidas preventivas bem como o tratamento de grupos e indivíduos de forma diferenciada.

Idosos acometidos pela COVID-19 podem estar mais suscetíveis à desnutrição. Isto pode ser decorrente da presença de sintomas gastrointestinais, apresentados por grande parte dos idosos infectados, que interferem diretamente no consumo alimentar e estado nutricional, ou pelos efeitos deletérios da resposta inflamatória aguda ao SARS-CoV-2. Um fator preocupante é que os sintomas gastrointestinais são frequentes em pacientes com rápida progressão e pior desfecho da doença. Assim como a desnutrição, o sobrepeso acarreta prejuízos para o sistema imune, aumentando a frequência e gravidade de doenças infecciosas, levando a um maior risco de hospitalização, doenças graves e mortalidade. Apesar disso, apenas a obesidade é apontada como fator de risco para a COVID-19 (LI T, et al., 2020; SIMONNET A, et al., 2020).

Desta forma, a manutenção do bom estado nutricional pode contribuir para redução de complicações decorrentes das comorbidades e da COVID-19. Pacientes infectados, do grupo de risco ou recuperados precisam de suporte nutricional adequado, devido as infecções virais estarem associadas a deficiências nutricionais, sendo importante a alimentação saudável para todos os idosos (BARAZZONI R, et al., 2020).

A presença da deficiência cognitiva mostrou-se com diferenças significantes na incapacidade, limitação física, percepção de saúde e vulnerabilidade total nos idosos participantes do estudo. O declínio cognitivo origina-se do próprio processo de envelhecimento devido ao avançar da idade e do acúmulo de diversas doenças. Associado a esses fatores, a institucionalização compromete ainda mais a capacidade cognitiva na pessoa idosa, fazendo com que se sinta isolado devido à ausência do suporte familiar, dificultado a realização das ABVD, bem como a redução da mobilidade e a uma percepção negativa de saúde (GUTHS JCFS, et al., 2017).

Idosos com declínio cognitivo podem sofrer um grande impacto na qualidade de vida frente à pandemia do COVID-19, pois, a perda da autonomia associada à deficiência cognitiva interfere diretamente no desempenho das atividades do cotidiano, limitando e/ou dificultando medidas de higiene, conforto e bem 
estar, fazendo-se necessária atenção diferenciada, fornecendo suporte adequado a este público. Além disso, as restrições de isolamento social adotadas como medida necessária com vistas às ações de combate a disseminação do vírus podem agravar esse impacto (CAMACHO ACLF, et al., 2020.)

O estudo demonstrou que a presença da hipertensão foi significante com os domínios de faixa etária, percepção de saúde e vulnerabilidade total. A Hipertensão Arterial Sistêmica (HAS) é uma doença que atinge em torno de $45 \%$ da população geral, com aumento significativo com o avançar da idade. Apesar da influência da HAS em patologias que afetam sistema respiratório, como no caso da COVID-19, ainda não ser totalmente esclarecida, estudos epidemiológicos indicam o aumento da mortalidade da população que apresenta associação desses fatores e afetam principalmente pacientes com 60 anos ou mais.

Dados obtidos por meio do Sistema de Informação de Doenças Infecciosas da China demonstraram que a HAS era uma das comorbidades que estava presente em pacientes com 60 anos ou mais que foram a óbito ou tiveram complicações pela COVID-19. De acordo com alguns estudos realizados, a relação da HAS com a COVID-19 pode ocorrer por fatores como, o uso de anti-hipertensivos específicos, apesar destes apresentarem algumas controvérsias nos resultados das análises dos estudos, ou pela própria fisiologia do indivíduo portador de HAS, que apresenta uma redução da capacidade respiratória (BARROS GM, et al., 2020).

Em um estudo retrospectivo realizado em dois hospitais de Wuhan, onde foram avaliados 191 doentes, foi identificado a prevalência de HAS em $48 \%$ dos indivíduos que foram a óbito, sendo observado ainda nesse estudo que o índice de internação em unidades de cuidados intensivos foi mais prevalente em pacientes que apresentavam hipertensão, diabetes e doença cardiovascular (ZHOU F, et al., 2020).

\section{CONCLUSÃO}

O IMC alterado, deficiência cognitiva e hipertensão foram considerados os fatores de risco para COVID19 mais importantes encontrados. Além disso, quando analisada a presença dos fatores de risco com a vulnerabilidade, os itens incapacidade, limitação e faixa etária apresentaram associação. Considerando os resultados encontrados, torna-se necessário destacar que idosos considerados com presença de vulnerabilidade estão mais susceptíveis a ter fatores de risco para COVID-19. Os idosos institucionalizados são o público de maior alvo de infecção por COVID-19, os resultados mostram que isso está inerente a presença dos aspectos de vulnerabilidade conforme observado. É preciso fortalecer o cuidado no que tange a presença de vulnerabilidade, dessa forma, avaliar os fatores de risco e promover estratégias para diminuição e controle desses agravos se configura como um importante indicador para minimizar os riscos de adoecer por COVID-19.

\section{AGRADECIMENTOS}

O projeto foi financiado pela Rede Internacional de Pesquisa Sobre Vulnerabilidade, Saúde, Segurança e Qualidade De Vida Do Idoso: Brasil, Portugal e Espanha (Edital 01/2020/UFRN) e Conselho Nacional de Desenvolvimento Científico e Tecnológico (CNPq) - pagamento de bolsa produtividade a Gilson de Vasconcelos Torres [processo 309213 / 2017-7].

\section{REFERÊNCIAS}

1. AGÊNCIA NACIONAL DE VIGILÂNCIA SANITÁRIA (ANVISA). Nota técnica n. 05/2020 GVIMS/GGTES. Orientações para a Prevenção e Controle de Infecções pelo novo coronavírus (SARS-Cov-2) em Instituições de Longa Permanência para Idosos (ILPI). Brasília, 24 de março de 2020. [Internet]. 2020. [acesso em 02 abr. 2020]. Disponível em: https://www20.anvisa.gov.br/segurancadopaciente/index.php/alertas/item/nota-tecnica-n-05-2020-gvims-ggtesanvisa-orientacoes-para-a-prevencao-e-o-controle-de-infeccoes-pelo-novo-coronavirus-sars-cov-2-ilpi

2. ARAUJO JR FB, et al. Fragilidade, perfil e cognição de idosos residentes em área de alta vulnerabilidade social. Revista Ciência \& Saúde Coletiva, 24(8):3047-3055, 2019.

3. BARBOSA KTF, et al. Envelhecimento e vulnerabilidade individual: um panorama dos idosos vinculados à estratégia saúde da família. Revista texto contexto enferm 2017; 26(2):e2700015.

4. BARBOSA IR, et al. Incidência e mortalidade por COVID-19 na população idosa brasileira e sua relação com indicadores contextuais: um estudo ecológico. Revista Brasileira de Geriatria e Gerontologia 2020; 23(1).

5. BARROS GM, et al. Considerações sobre a relação entre a hipertensão e o prognóstico da COVID-19. J Health Biol Sci. $2020 \mathrm{~J} ; 8(1): 1-3$. 
6. BARAZZONI R, et al. ESPEN expert statements and practical guidance for nutritional management of individuals with SARS-CoV-2 infection. Clin Nutr. 2020 39(6):1631-38.

7. BENKSIM A, et al. VulnerabilityandFragilityExposeOlder-AdultstothePotentialDangersof COVID-19 Pandemic. Iran J Public Health 2020; 49(Suppl.1): 122-124.

8. BRASIL, Ministério da Saúde. Caderneta de Saúde da Pessoa Idosa. Brasília: Editora do Ministério da Saúde, 2013. Disponível em: http://portalms.saude.gov.br/saude-paravoce/saude-da-pessoa-idosa/caderneta-da-saude-da-pessoaidosa. Acesso em: 22/11/2019.

9. BROOKE J, DEBRA J. Olderpeopleand COVID-19: Isolation, riskandageism. JournalClinical Nursing 2020; 00:1-3.

10. CAMACHO ACLF, et al. de. Tecnologia da informação ao idoso em tempos de COVID-19. Research, Society and Development 2020; 9(6): e124963497, 2020 (CC BY 4.0).

11. CRUZ RR, et al. Envelhecimento e vulnerabilidade: análise de 1.062 idosos. Rev. Bras. Geriatr. Gerontol. 2019; 22(3):e180212..

12. GUTHS JCFS, et al. Perfil sociodemográfico, aspectos familiares, percepção de saúde, capacidade funcional e depressão em idosos institucionalizados no Litoral Norte do Rio Grande do Sul, Brasil. Rev. Bras. Geriatr. Gerontol $2017 ; 20(2): 175-185$.

13. JESUS IT, et al. Fragilidade de idosos em vulnerabilidade social. Revista Acta Paul Enferm. 2017; 30(6):614-20.

14. LI T, et al. Prevalence of malnutrition and analysis of related factors in elderly patients with COVID-19 in Wuhan, China. Eur J Clin Nutr. 2020 Disponível em: http://dx.doi.org/10.1038/s41430-020-0642-3

15. LIMA DLF, et al. COVID-19 no estado do Ceará, Brasil: comportamentos e crenças na chegada da pandemia. Ciência \& Saúde Coletiva 2020; 25(5):1575-1586.

16. LLOYD-SHERLOCK P, et al. Bearing the brunt of COVID-19: older people in low and middle income countries. MJ 2020; 368:m1052.

17. MINISTÉRIO DA SAÚDE (BR). Secretaria de Vigilância em Saúde. Boletim Epidemiológico. Infecção Humana pelo Novo Coronavírus (2019-nCoV). [Internet]. Brasília: Ministério da Saúde; 2020. [acesso em 31 mar 2020]. Disponível em: https://www.saude.gov.br/images/pdf/2020/fevereiro/04/Boletim-epidemiologicoSVS-04fev20.pdf.

18. NUNES VM et al. COVID-19 e o cuidado de idosos: recomendações para instituições de longa permanência. Natal: EDUFRN; 2020. [Internet]. 2020.

19. OPAS, Organização Pan-Americana de Saúde (2020). Folha informativa- CO-VID-19 (doença causada pelo novo $\begin{array}{lllll}\text { coronavírus). } & \text { Acesso } & \text { em } & \text { de } & \text { maio, }\end{array}$ https://www.paho.org/bra/index.php?option=com_content\&view=article\&id=6101: covid19\&ltemid=875

20. OXFORD COVID-19 EVIDENCE SERVICE. Global COVID-19 case fatalityrates. 2020. Disponível em: https://www.phc.ox.ac.uk/COVID-19/evide nceservice/revie ws/global-COVID-19-case-fatal ityrates

21. PEREIRA FM, et al. Recomendações dos serviços de geriatria e cuidados paliativos do hospital das clínicas da ufmg para manejo (acolhimento e classificação) do idoso e adulto com comorbidade grave com infecção pelo coronavírus. Linha De Cuidados Paliativos Do Hc Ufmg - Equipe De Cuidados Paliativos Adulto Do Hc Ufmg. Núcleo De Geriatria E Gerontologia Do Hc Ufmg. Belo Horizonte, 01 de abril de 2020.

22. PETRILLI CM, et al. Factors associated with hospitalization and critical illness among 4,103 patients with Covid-19 disease in New York City. BMJ. 2020; 369:m1966.

23. SCHMIDT TP. Padrões de multimorbidade e incapacidade funcional em idosos brasileiros. Universidade Federal De Santa Catarina Campus Araranguá Departamento De Ciências Da Saúde Curso De Fisioterapia. Araranguá/SC 2019.

24. SILVA RS, et al. Condições de saúde de idosos institucionalizados: contribuições para ação interdisciplinar e promotora de saúde. Cad. Bras. Ter. Ocup. 2019; 27(2): 345-356.

25. SIMONNET A, et al. High prevalence of obesity in severe acute respiratory syndrome coronavirus-2 (SARS-CoV-2) requiring invasive mechanical ventilation. Obesity (Silver Spring). 2020. Disponível em: https://doi.org/10.1002/oby.22831

26. TAN LF e SEETHARAMAN SK. COVID-19 outbreak in nursing homes in Singapore. J Microbiol Immunol Infect 2020 [Epub ahead of print]. Disponível em: https://doi.org/10.1016/j.jmii.2020.04.018

27. ZHANG W. Manual de Prevenção e Controle da COVID-19 segundo o Doutor Wenhong Zhang. São Paulo: Polo Books; 2020. 3. Ministério da Saúde (BR). O que é o Coronavírus? (COVID-19). 2020.

28. ZHOU F, et al. Clinical course and risk fac-tors for mortality of adult inpatients with COVID-19 in Wuhan, China: a retrospective cohort study. Lancet; 2020; pii:S0140-6736(20)30566-3. 